\title{
Linee guida per la calcolosi: commento
}

\author{
Commento all'articolo: "Medical Management to prevent recurrent nephrolithiasis in adults: \\ A systematic review for an American College of Physicians. Clinical Guideline”
}

\section{Luca Dello Strologo}

Dipartimento di Nefrologia-Urologia, Ospedale Bambino Gesù, Roma

La calcolosi urinaria è una patologia frequente nella popolazione generale. In un'interessante metanalisi, "Medical management to prevent recurrent nephrolithiasis in adults: a systematic review for an American College of Physicians. Clinical Guideline" recentemente pubblicata in Annals of Internal Medicine (1), vengono riprese criticamente le indicazioni della letteratura circa i comportamenti generalmente suggeriti dai clinici per ridurre il rischio di recidiva della calcolosi.

Per questo scopo, sono stati selezionati 28 studi randomizzati. In 23 studi erano considerati pazienti con calcolosi esclusivamente a base calcica, in 3 casi pazienti con calcoli di struvite e in 2 erano inclusi pazienti con varie tipologie di calcoli. In 8 casi era preso in esame l'effetto delle prescrizioni dietetiche e in 20 l'effetto di interventi farmacologici.

Ne emergono alcune considerazioni interessanti.

Per quanto attiene alla valutazione delle prescrizioni dietetiche, si è confermato (e non sorprende) che l'aumento dell'apporto di liquidi e la riduzione dell'apporto di calcio riduce significativamente il rischio di recidiva, almeno con riferimento ai soggetti con calcolosi di tipo calcico.

Altro dato interessante è il minor numero di recidive dopo riduzione dell'assunzione di bevande analcoliche gassate, cosìddette "soft drinks". Questo appare evidente, in realtà, prevalentemente se ci si riferisce alla riduzione di quelle acidificate con acido fosforico (bevande a base di cola).

Poco evidente era invece l'effetto protettivo dell'apporto di fibre e la riduzione del consumo delle proteine animali.

Rispetto all'uso di terapie farmacologiche, l'uso di alcuni preparati (tiazidici, allopurinolo, citrato e N-idrossiacetamide) è apparso discretamente efficace nella prevenzione delle recidive di calcolosi. Tuttavia, sulla base degli studi disponibili, il livello di raccomandazione rispetto a queste prescrizioni non può essere definito "forte".

In un numero molto limitato di studi, è stata anche studiata la possibilità di predire l'efficacia del trattamento analizzando i dati biochimici del paziente pre-trattamento. Se si escludono casi particolari, nei quali erano presenti più anomalie biochimiche contemporaneamente, l'analisi biochimica non era contributiva. In particolare, la presenza di ipercalciuria non è risultata significativamente associata con l'efficacia di una terapia profilattica. Forse un poco più utile il monitoraggio dell'escrezione urinaria di acido urico, ove coinvolto nella genesi della calcolosi.

Una valutazione critica di questi dati è necessaria.

La maggior parte dei pazienti inclusi negli studi presentavano calcoli di ossalato di calcio. Questo è naturale, perché rappresenta la tipologia di calcolosi di gran lunga più frequente. Tuttavia, non sarebbe corretto esportare automaticamente i risultati osservati ad altri tipi di calcolosi. In particolare, non è certo possibile considerare i risultati applicabili a patologie, come ad esempio la cistinuria, per le quali il rischio di recidiva è elevatissimo (2) e per le quali è indispensabile un trattamento che incida direttamente sulle cause (chelanti della cistina, alcalinizzazione) (3).

Qualunque sia stato l'intervento impostato nei vari studi (farmacologico, dietetico ecc.), tutti i pazienti venivano comunque istruiti ad aumentare l'apporto di liquidi. Questa pratica è chiaramente molto efficace ed è possibile che possa avere un effetto così forte da mascherare l'efficacia delle altre misure. Per la maggior parte di queste, in effetti, il grado di raccomandazione appare modesto.

Numerosi altri fattori probabilmente concorrono a questa circostanza: il numero di pazienti arruolabile non è elevato, la costanza nel tempo dell'aderenza alle indicazioni fornite è spesso incerta e, soprattutto, il basso numero di recidive atteso nella maggior parte dei casi, rende difficile comprendere appieno l'effetto protettivo di qualunque terapia. Per ottenere una riduzione significativa dei nuovi eventi calcolotici, infatti, sarebbe necessario arruolare un enorme numero di pazienti che andrebbero seguiti per un tempo lungo e, in una patologia non-vita-minacciante e, tutto sommato, scarsamente invalidante, questo è difficile.

La riduzione dell'assunzione di "soft drinks" può certamente avere un effetto in soggetti che ne assumano ingenti quantitativi, ma nella nostra cultura, l'uso di queste bevande è spesso più limitato che in altri contesti, anche se probabilmente i soggetti più giovani possono in alcuni casi farne largo uso.

Dall'analisi dell'intero articolo sembra comunque potersi evincere che il trattamento più efficace comprende numerosi interventi. Certamente il ruolo fondamentale è giocato dall'incremento dell'apporto di fluidi e dalla conseguente prevenzione di periodi di disidratazione/sottoidratazione. Su questo aspetto non ci sono dubbi e va certamente perseguito, ricordando anche ai pazienti che la mancata assunzione di liquidi nel periodo notturno può facilitare la comparsa di calcoli renali. Anche la riduzione del sodio 
nella dieta appare efficace nelle calcolosi a base calcica e/o ossalica.

La riduzione dell'assunzione di bevande gassate acidificate con acido fosforico è anche da suggerire.

L'uso di farmaci in grado di aumentare la solubilità (citrati) o ridurre la concentrazione dei singoli soluti (tiazidici e allopurinolo) rappresenta un ulteriore ausilio e deve essere parte integrante delle prescrizioni del clinico.

Altre prescrizioni dietetiche non sembrano invece avere un effetto significativo e non trovano quindi giustificazione.

Da notare che queste conclusioni sono state confermate da una recentissima review Cochrane (4) e dovrebbero ormai essere considerate parte integrante delle nostre prescrizioni.

Dichiarazione di conflitto di interesse: L'Autore dichiara di non avere conflitto di interessi.

Contributi economici agli Autori: L'Autore dichiara di non avere ricevuto sponsorizzazioni economiche per la preparazione dell'articolo.

Indirizzo dell'Autore:

Dr. Luca Dello Strologo

Dipartimento di Nefrologia Urologia

Ospedale Pediatrico Bambino Gesù

Piazza S. Onofrio 4

00165 Roma

luca.dellostrologo@opbg.net

\section{Bibliografia}

1. Fink HA, Wilt TJ, Eidman KE, et al. Medical management to prevent recurrent nephrolithiasis in adults: a systematic review for an American College of Physicians Clinical Guideline. Ann Intern Med 2013; 158(7): 535-43.

2. Dello Strologo L, Pras E, Pontesilli C, et al. Comparison between SLC3A1 and SLC7A9 cystinuria patients and carriers: a need for a new classification. J Am Soc Nephrol 2002; 13(10): 2547-53.

3. Dello Strologo L, Laurenzi C, Legato A, Pastore A. Cystinuria in children and young adults: success of monitoring free-cystine urine levels. Pediatr Nephrol 2007 22(11): 1869-73.

4. Escribano J, Balaguer A, Roqué I, Figuls M, Feliu A, Ferre N. Dietary interventions for preventing complications in idiopathic hypercalciuria. Cochrane Database Syst Rev 2014; 2: CD006022. 